\title{
Aspectos fundamentales para la acreditación de laboratorios de calibración de equipos médicos en Colombia
}

\section{Fundamental aspects for accrediting medical equipment calibration laboratories in Colombia}

\author{
Luis E. Llamosa-Rincón ${ }^{1}$, Giovanni A. López-Isaza ${ }^{2}$ y Milton F. Villarreal-Castro ${ }^{3}$ \\ 1 Facultad de Ciencias Básicas, Departamento de Física. Universidad Tecnológica de Pereira, \\ Colombia.1ellamo@utp.edu.co \\ 2 Facultad de Tecnologías, Escuela de Tecnología Industrial. Universidad Tecnológica de Pereira, \\ Colombia.galijamj@yahoo.es, galijamj@gmail.com \\ 3 Laboratorio de Metrología Electromédica - Universidad Tecnológica de Pereira, Colombia. \\ milfer@utp.edu.co
}

Recibido 6 Junio 2009/Enviado para Modificación 21 Enero 2010/Aceptado 4 Febrero 2010

\section{RESUMEN}

Objetivo Analizar los aspectos metrológicos fundamentales que se deben tener en cuenta en la elaboración de los procedimientos de calibración de equipo electromédico, que permitan acreditar en Colombia, laboratorios de metrología electromédica con base en la normatividad internacional.

Métodos Con base en las normas NTC-ISO-IEC 17025:2005 y GTC-51, se diseñaron e implementaron procedimientos para la calibración de equipo electromédico, contando para ello con un conjunto completo de analizadores de equipos electromédicos que fueron utilizados como patrones.

Resultados El modelo matemático para determinar el valor estimado de incertidumbre en la calibración de equipos electromédicos, para la acreditación del Área de Calibración de Equipos Electromédicos del Laboratorio de Metrología de Variables Eléctricas, acreditado según Resolución 25771 del 26 mayo de 2009 de la Superintendencia de Industria y Comercio, consta de dos ecuaciones según sea el caso; ellas son: $\mathrm{E}=(\overline{\mathrm{Ai}}+\overline{\mathrm{DA}})-(\mathrm{Ar}+\bar{\delta} \mathrm{Ar}+\delta A r 1)$ y $\mathrm{E}=(\mathrm{Ai}+\bar{\delta} \mathrm{Ai})-(\overline{\mathrm{Ar}}+\overline{\mathrm{D}} \mathrm{Ar}+\delta A r \mathrm{c})$ Conclusión Eı moaeiamiento matematıco ımplementaao para el calcuio de incertidumbre de medición en el proceso de acreditación del Área de calibración de equipos electromédicos del Laboratorio de Metrología de Variables Eléctricas, de la Universidad Tecnológica de Pereira, constituye una buena guía para futuros procesos de calibración que emprendan otros laboratorios de Colombia y Latinoamérica.

Palabras Clave: Acreditación, calibración, seguridad de equipos, incertidumbre (fuente: DeCS, BIREME).

\begin{abstract}
Objective Analysing the fundamental methodological aspects which should be considered when drawing up calibration procedure for electro-medical equipment, thereby
\end{abstract}


permitting international standard-based accreditation of electro-medical metrology laboratories in Colombia.

Methods NTC-ISO-IEC 17025:2005 and GTC-51-based procedures for calibrating electro-medical equipment were implemented and then used as patterns.

Results The mathematical model for determining the estimated uncertainty value when calibrating electro-medical equipment for accreditation by the Electrical Variable Metrology Laboratory's Electro-medical Equipment Calibration Area accredited in compliance with Superintendence of Industry and Commerce Resolution 25771 May $26^{\text {th }}$ 2009 consists of two equations depending on the case; they are:

$E=(\overline{A i}+\delta A i)-(A r+\delta A r+\delta A r 1)$ and $E=(A i+\delta A i)-(\overline{A r}+\delta A r+\delta A r 1)$

Conclusion The mathematical modelling implemented tor measurıng uncertainty in the Universidad Tecnológica de Pereira's Electrical Variable Metrology Laboratory (Electro-medical Equipment Calibration Area) will become a good guide for calibration initiated in other laboratories in Colombia and Latin-America.

Key Words: Accreditation, calibration, equipment safety, uncertainty (source: $\mathrm{MeSH}$, $N L M)$.

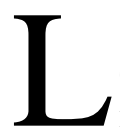

a acreditación es un procedimiento mediante el cual se reconoce la competencia técnica y la idoneidad de organismos de certificación e inspección, laboratorios de ensayo y laboratorios de metrología para que lleven a cabo las actividades a las que se refiere el decreto 2269 de 1993 y reglamentadas en la Norma Técnica Colombiana NTC-ISO-IEC 17025 (1).

Con base en la experiencia en el proceso de acreditación del primer laboratorio que presta servicios de calibración para equipo electromédico, acreditado en Colombia, según resolución 25771 del 26 mayo de 2009 de la Superintendencia de Industria y Comercio SIC, por una parte, y teniendo en cuenta estudios realizados en instituciones prestadoras de servicios de salud, en los que se identificó que uno de los factores que pone en peligro el proceso de certificación, es la calibración de equipos electromédicos $(2,3)$, se presenta un análisis acerca de los aspectos metrológicos fundamentales que deben ser implementados dentro de los procedimientos de calibración de un laboratorio de metrología electromédica -LME-, para que pueda ser "acreditado" con base en la norma NTC-ISO/IEC 17025:2005. Se incluye como resultado principal, el diseño implementado para el cálculo de incertidumbre de medición de los procedimientos de calibración respectivos, ya que éste es uno de los aspectos de mayor importancia y es donde existen mayores dudas en el momento de implementarlos. 


\section{MÉTODOS}

En este trabajo se utilizan las definiciones metrológicas de conformidad con la norma NTC-2194 (4) y la norma NTC-IEC-60601-1 (5), además en lo que respecta al cálculo de incertidumbre de medición se utiliza la norma GTC-51 (6) "Guía para la expresión de incertidumbre en las mediciones" que es equivalente con la "Guide to the expression of uncertainty in measurement" publicada por la ISO. Los aspectos metodológicos y metrológicos fundamentales para la acreditación de laboratorios de calibración de equipo electromédico se basan en la norma NTC-ISO-IEC-17025 (1). Para que un laboratorio de calibración/ensayo logre pertenecer al Sistema Nacional de Normalización Certificación y Metrología (SNNCM) debe someterse voluntariamente a un proceso de acreditación ante la Superintendencia de Industria y Comercio- SIC. Además de los documentos de gestión, un LME debe tener los documentos pertinentes para realizar calibraciones, lo anterior con el fin de cumplir con los numerales de la 17025: 5.4 Métodos de ensayo y calibración y validación de métodos y 5.10 Reporte de resultados. Lo anterior se cumple, mediante el empleo de los siguientes documentos: Procedimientos, Instructivos de trabajo, documentos técnicos y formatos, dentro de los cuales, los aspectos metrológicos en especial el cálculo de incertidumbre de medición son de fundamental importancia.

La metodología utilizada para implementar un procedimiento genérico para el cálculo de incertidumbre de medición para los procedimientos de calibración de equipo electromédico, parte del hecho que se relaciona con que ellos se pueden clasificar en dos grandes grupos: Los que miden una variable y aquellos que la generan. Esto hace que existan dos procedimientos muy similares que parten de un modelamiento matemático particular.

\section{RESULTADOS}

Uno de los aspectos científicos de mayor importancia, en el que tiene mayores dudas el personal técnico de un laboratorio en el proceso de realizar calibración de equipos electromédicos, es el cálculo de incertidumbre de medición. Dada su pertinencia e importancia en el proceso de acreditación, se describe el procedimiento diseñado para determinar el valor estimado de la incertidumbre y lo correspondiente a la expresión de su resultado en la calibración de equipos electromédicos. Se pueden presentar dos casos:

Si el equipo electromédico para calibración -EEC-mide la variable y el patrón de trabajo la genera, se desarrolla el siguiente procedimiento: 
- Modelar matemáticamente el procedimiento de medición

$$
E=(\overline{A i}+\delta A i)-(A r+\delta A r+\delta A r 1)[1]
$$

Donde: Ar (lectura del patrón de trabajo, $\overline{A i}$ (valor medio de las lecturas del equipo a calibrar), $\delta \operatorname{Ar}$ (corrección en la indicación del patrón de trabajo debido a: Desviaciones en la Temperatura ambiente y humedad relativa; variaciones en la fuente de alimentación; variaciones a partir de calibraciones pasadas), $\delta A \mathrm{i}$ (corrección de indicación por resolución del equipo a calibrar), $\delta \mathrm{Ar}_{1}$ (corrección de indicación por resolución del patrón de trabajo), E (error absoluto).

Aclaraciones a la ecuación [1]: La resolución corresponde al dígito menos significativo observado en la pantalla del equipo a calibrar o el patrón de trabajo. La corrección en la indicación por resolución es igual a cero (numeral S 9.7 EA 4/02) (7), por lo tanto: $\delta A \mathrm{i}=\delta \mathrm{Ar}_{1}=0,0$. Para la corrección en la indicación del patrón de trabajo, se tiene en cuenta los aspectos establecidos por el fabricante, el patrón de trabajo se calibra cada año y además el resultado del certificado de calibración muestra que las especificaciones del fabricante se cumplen, el estimado de $\delta \operatorname{Ar}$ es 0,0 . De acuerdo con lo anterior la ecuación [1] se reduce a:

$$
\mathrm{E}=\overline{\mathrm{Ai}}-\mathrm{Ar} \text { [2] }
$$

- Evaluar los tipos de incertidumbre estándar. En los tipos de incertidumbre (Tipo A y tipo B) se presentan diferentes fuentes de incertidumbre durante el proceso de medición (GTC-51 numeral 3.3.2) (6), esas fuentes se visualizan en la Figura 1:

- Evaluar la incertidumbre Tipo A por repetibilidad de las lecturas (GTC-51 numeral 4.2.3) (6).

Figura 1. Fuentes de incertidumbre involucradas en el proceso de medición

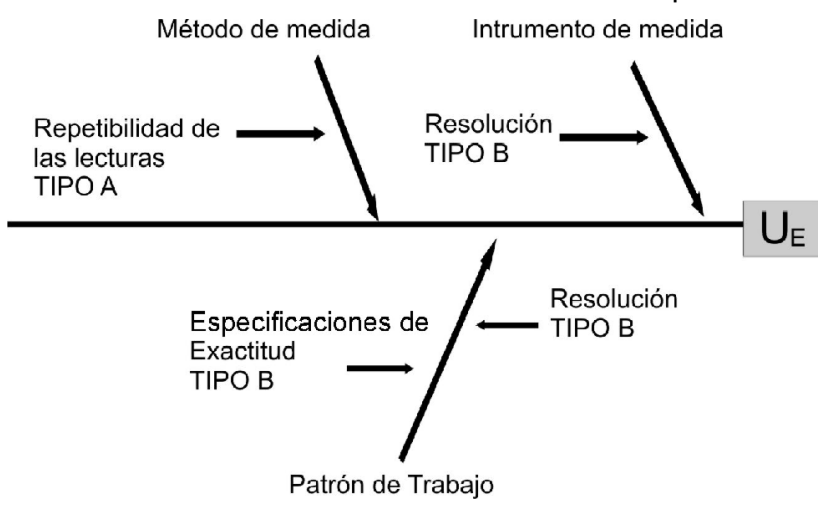




$$
\overline{A i}=\frac{1}{n} * \sum_{i=1}^{n} A i i \quad[3] \quad S(\overline{A i})=\sqrt{\frac{1}{n-1} * \sum_{i=1}^{n}(A i i-\overline{A i})^{2}} \quad \text { [4] } \quad U_{A}=\frac{S(\overline{A i})}{\sqrt{n}} \quad[5]
$$

Donde: Aii (cada una de las lecturas del equipo registradas durante la calibración), $\mathrm{n}$ (número de mediciones realizadas en la calibración; este número puede ser 3 por la repetibilidad de los instrumentos), S(Ai) (desviación estándar), $\mathrm{U}_{\mathrm{A}}$ (incertidumbre estándar tipo A). Se continúa con los siguientes pasos:

- Evaluar la incertidumbre Tipo B por especificaciones de exactitud del patrón de trabajo (UB1) (GTC-51 numeral 4.3)(6)-distribución rectángular. - Evaluar la incertidumbre Tipo B por resolución del patrón de trabajo (UB2) - distribución rectángular. - Evaluar la incertidumbre Tipo B por resolución del equipo a Calibrar (UB3) - distribución rectángular.

- Hallar los coeficientes de sensibilidad (GTC-51 numeral 5.1.4/ 5) (6). Partiendo de la ecuación [2] obtenemos [9].

- Determinar el valor de la incertidumbre combinada UC (GTC51 numeral 5.1.2) (6). Esta incertidumbre se calcula a partir de $\mathrm{U}_{\mathrm{B} 1}=\frac{\text { Especificaciones del patrón de trabajo }}{\sqrt{3}}[6]$

$$
\mathrm{U}_{\mathrm{B} 2}=\frac{\text { Resolución }}{2 * \sqrt{3}}
$$

$$
\mathrm{U}_{\mathrm{B} 3}=\frac{\text { Resolución }}{2 * \sqrt{3}}[8]
$$

$$
\frac{\partial \mathrm{E}}{\partial \overline{\mathrm{Ai}}}=1 ; \quad \frac{\partial \mathrm{E}}{\partial \mathrm{Ar}}=-1 \quad[9]
$$
las incertidumbres estándar (Tipo A y Tipo B) y de los coeficientes de sensibilidad (ecuación 9).

$$
\begin{aligned}
& U_{C}=\sqrt{\left(\frac{\partial E}{\partial \bar{A}}\right)^{2} \times U_{A}^{2}+\left(\frac{\partial E}{\partial A r}\right)^{2} \times\left(U_{B 1}^{2}+U_{B 2}^{2}\right)+\left(\frac{\partial \mathrm{E}}{\partial \bar{A} \mathrm{i}}\right)^{2} \times U_{B 3}^{2}} \\
& U_{C}=\sqrt{U_{A}^{2}+U_{B 1}^{2}+U_{B 2}^{2}+U_{B 3}^{2}}
\end{aligned}
$$

- Identifique la incertidumbre dominante en la ecuación [10] (EA-4-02 numeral S9.11/14) (7).

- Calcule la incertidumbre combinada sin tener en cuenta la incertidumbre

$$
\mathrm{U}_{\mathrm{C}}^{\prime}=\sqrt{U_{C}^{2}-U_{\text {Domin ante }}^{2}}
$$
estándar dominante.

- Calcule la relación entre $\mathrm{U}_{\mathrm{C}} \mathrm{y} \mathrm{U}_{\text {Dominante. }}$

$$
\mathrm{C}=\mathrm{U}_{\mathrm{C}}^{\prime} / \mathrm{U}_{\text {Dominante }}[12]
$$


- ¿La relación de la ecuación [12] es menor a 0,3? a) Si la relación es menor a 0,3 , siga las actividades marcadas con (*). b) Si la relación es mayor o igual a 0,3 , siga las actividades marcadas con $(* *)$.

- * De acuerdo con el ítem a), asuma un factor de cobertura $\mathrm{k}=1,65$.

- *Calcule la incertidumbre expandida (GTC-51 numeral 6.2.1) (6) de acuerdo con la siguiente expresión: $U_{\mathrm{E}}=\mathrm{k} \mathrm{U}_{\mathrm{C}}=1,65 \mathrm{U}_{\mathrm{C}} \quad$ [13]

- ** De acuerdo con el enunciado b), halle el número de grados efectivos de

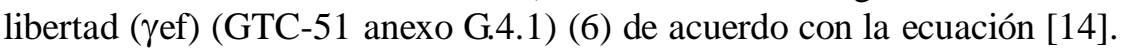

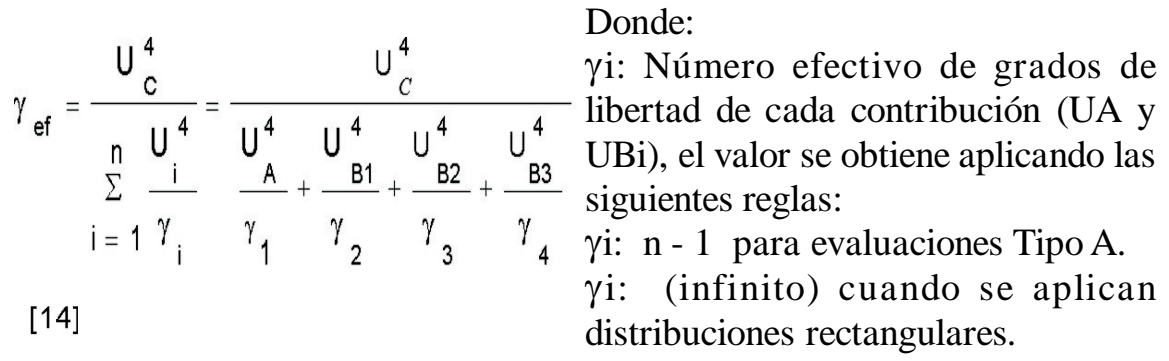

- ** Teniendo en cuenta un nivel de confianza del $95 \%$ y el valor obtenido de la ecuación [14], defina el factor de cobertura k de Student en función del número efectivo de grados de libertad.

- ** Calcule la Incertidumbre Expandida de acuerdo con la ecuación [15].

$$
\mathrm{UE}=\mathrm{U}_{\mathrm{C}} \quad \mathrm{k}[15]
$$

Si el -EEC- genera la variable y el patrón de trabajo la mide, se desarrolla el mismo procedimiento anterior con algunas modificaciones que se expresan en las definiciones de los componentes de la ecuación [16] (modelamiento matemático del procedimiento de medición) que a continuación se presenta:

$$
E=(A i+\delta A i)-(\overline{A r}+\delta A r+\delta A r l)[16]
$$

Donde: $\overline{\mathrm{Ar}}$ (valor medio de las lecturas del equipo patrón), Ai (Lectura del equipo a calibrar), $\delta A r$ (corrección en la indicación del patrón de trabajo debido a: Desviaciones en la Temperatura ambiente y humedad relativa; variaciones en la fuente de alimentación; variaciones a partir de calibraciones pasadas), $\delta$ Ai (corrección de indicación por resolución del equipo a calibrar), 
$\delta \mathrm{Ar}_{1}$ (corrección de indicación por resolución del patrón de trabajo), E (error absoluto). De la expresión [16] se pueden hacer las mismas aclaraciones que se realizaron para la expresión [1] en cuanto a la resolución y en cuanto a la corrección en la indicación del patrón de trabajo.

De acuerdo con lo anterior la ecuación [16] se reduce a: $\mathrm{E}=A i-\overline{\mathrm{Ar}} \quad[17]$

A continuación se sigue el mismo procedimiento seguido para el caso en que el equipo electromédico para calibración mide la variable y el patrón de trabajo la genera.

Expresión de resultados: El valor de la incertidumbre de medida se expresa con dos cifras significativas, el número de cifras significativas para Error (E), Tolerancia (T), lecturas en el equipo a Calibrar (Ai) y las lecturas del analizador (Ar), equivale a aumentar una cifra más que la observada en el valor de resolución de los equipos. Esta consideración hace que la medida realizada sea más exacta, se evita la sobre-estimación de valores por causa de aproximaciones, lo cual afectaría por especificaciones a los equipos electromédicos. Para realizar las aproximaciones o redondeos de los datos, tener en cuenta lo siguiente: Si el dígito siguiente a la última cifra significativa es mayor o igual a 5, la última cifra significativa se incrementa en 1 , de lo contrario no se modifica.

Análisis de resultados. El análisis de resultados que permite verificar si un instrumento se encuentra dentro o fuera de las especificaciones con que fue comprado en lo que respecta su a tolerancia $(\mathrm{T})$ reportada por el fabricante se basa en los siguientes criterios:

Si el -EEC- mide la variable y el patrón de trabajo la genera:

Instrumento de medida Dentro de especificaciones:

$$
|\overline{A i}-A r|<[T|-| U e \mid]
$$

Instrumento de medida Fuera de especificaciones:

Indeterminacion:

$$
|A \bar{i}-A r|>[T|+| U e \mid]
$$

$$
[T|-| U e \mid]|\overline{A i}-A r|<[T|+| U e \mid]
$$


Donde $\overline{\mathrm{Ai}}$ es el promedio de las lecturas del instrumento electromédico que se calibra y $A r$ el valor generado por el instrumento patrón.

Si -EEC- genera la variable y el patrón de trabajo la mide:

Instrumento de medida Dentro de especificaciones:

$$
|A i-\overline{A r}|<[T|-| U e \mid]
$$

Instrumento de medida Fuera de especificaciones:

$$
|A i-\overline{A r}|>[T|+| U e \mid]
$$

Indeterminación:

$$
[T|-| U e|k| A i-\overline{A r} \mid<[T|+| U e \mid]
$$

Donde $A i$ es el valor generado por el instrumento electromédico que se calibra y $\overline{\mathrm{Ar}}$ el promedio de la lecturas del instrumento patrón. Se considera que un instrumento que reporta resultados indeterminados se debe considerar fuera de especificaciones dentro del rango analizado.

\section{DISCUSIÓN}

La salud pública requiere de estándares para el seguimiento y transparencia en la prestación de servicios de salud, de forma que permita ganar la confianza de los usuarios, mejorar la imagen, caracterizar los equipos y procesos utilizados en las relaciones entre las entidades de salud y sus usuarios, favorecer la comercialización de sus servicios, la comunicación y la percepción de seguridad en la población de interés (2). La prestación de servicios de salud pública requiere de la gestión de un sistema de calibración de equipos contra patrones internacionales, de forma que los LME alcancen la acreditación en conformidad con estándares internacionales por una parte y por la otra, niveles de competitividad internacional.

Los aspectos metrológicos fundamentales para la acreditación de laboratorios que realicen calibración de equipo electromédico con base en la norma NTC-ISO-IEC-17025 son de especial importancia en el momento de elaborar procedimientos de calibración que permitan a un laboratorio acreditarse bajo esta norma; los procedimientos diseñados para el calculo de incertidumbre en los procesos de calibración de equipo electromédico con 
base en la norma GTC-51, equivalente a la "Guide to the expression of uncertainty in measurement" publicada por la ISO; y el éxito alcanzado en el proceso de acreditación del Laboratorio de Metrología Electromédica de la UTP, hacen que este trabajo sea un referente para futuros procesos de acreditación de laboratorios de calibración de equipo electromédico en Colombia y en Latinoamérica, para los cuales la cultura de la metrología y calidad, está hasta ahora entrando en todos los ámbitos, tanto académicos como de producción y de servicios

Agradecimientos: A COLCIENCIAS y a la Vicerrectoría de Investigaciones, Innovación y Extensión de la Universidad Tecnológica de Pereira, por la cofinanciación del proyecto, desarrollado por los grupos de investigación de Electrofisiología y sobre las capacidades tecnológicas de las organizaciones-GICTO, denominado "Evaluación de sistemas de Salud en lo referente a su capacidad tecnológica relacionada con la trazabilidad del equipo biomédico y su Seguridad eléctrica" (Contrato 443-2004, Código 1110-04-16430, PNCTS).

\section{REFERENCIAS}

1. Instituto Colombiano de Normas Técnicas y Certificación. Norma NTC-ISO-IEC 17025:2005 Requisitos generales de competencia de laboratorios de ensayos y calibración. Bogotá DC: ICONTEC;2002.

2. López-Isaza GA. Llamosa-Rincón, LE.Diagnóstico de la calibración del equipo biomédico en entidades de salud del Departamento de Risaralda. Rev. salud pública [Bogotá]. 2008; 10 (3): 462-469.

3.López-Isaza GA. Llamosa-Rincón, LE. Estudio de las capacidades tecnológicas relacionadas con la trazabilidad del equipo electromédico y su seguridad eléctrica en entidades de salud del Departamento de Risaralda; 2007.

4. Instituto Colombiano de Normas Técnicas y Certificación. Norma NTC-ISO-2194 Vocabulario de términos básicos y generales en metrología. Bogotá DC: ICONTEC; 2000.

5. Instituto Colombiano de Normas Técnicas y Certificación. Norma NTC-IEC-60601-1 Equipo electromédico. Parte 1: Requisitos generales para la seguridad. Bogotá DC: ICONTEC; 2000.

6. Instituto Colombiano de Normas Técnicas y Certificación.Norma GTC 51, Guía para la Expresión de Incertidumbre en Mediciones. Bogotá DC: ICONTEC; 2000.

7. EA-4/02 (European co-operation for accreditation) Expression of the Uncertainty of Measurement in Calibration; 1999. 